COMMENT

DOI: $10.1038 / \mathrm{s} 41467-018-07221-\mathrm{x}$

\title{
Prebiotic nucleic acids need space to
} grow

\author{
Daniel Whitaker ${ }^{1} \&$ Matthew W. Powner (i) ${ }^{1}$
}

What were the conditions on early Earth when nucleotides were formed, and what are the most plausible nucleoside candidates? Answering these questions will require mechanistic chemistry and planetary science to work together, enhancing not limiting each other's scope of investigation.

RNA is the leading candidate for life's first information-carrying biopolymer because of its dual role in information transfer and biocatalysis, as well as the deep-seated evolutionary history of non-coding RNAs. It is currently unknown when ribonucleosides first formed on Earth, but at some point between the formation of a stable hydrosphere 4.4 billion years ago $(4.4 \mathrm{Ga})^{1}$ and the emergence of life's last universal common ancestor ${ }^{2}$, RNA (and DNA) must have formed and been harnessed by life.

The limited evidence available for Earth's earliest history allows only broad-brush geochemical pictures to be considered. However, any discussion of the emergence of nucleosides on Earth must be tempered by consideration of what is prebiotically plausible. There is no definitive catalogue of (geo)chemical reactions that would have occurred on the Earth before the advent of life, and, even if there were, it is likely that most abiotic reactions would not play a significant role in initiating life. Clearly, some restrictions to standard organic chemistry must be made for a reaction to be considered prebiotically plausible. Orgel proposed that for a reaction run in a laboratory to qualify as prebiotic it must:

- use starting materials that could have been present in adequate amounts;

- proceed in water or without solvent;

- form the relevant product in a significant yield ${ }^{3}$.

This flexible definition allows a broad range of chemical reactions to be investigated without losing sight of the end goal-discovering the reactions that led to life. Although liberal, these constraints are informative and, relative to modern synthetic practices, highly restrictive. Limiting chemical reactions to those that proceed in water is geochemically reasonable, as water is the most abundant solvent on the Earth (5/7th of the Earth's surface is water-covered). However, chemically this limitation is massively restrictive and extremely challenging due to the low solubility of many organic molecules in water, the limited range of acidity and basicity available in water compared to organic solvents, as well as the potential for water itself to react as a competing nucleophile. On the other hand, the essential biochemical components which life uses are predisposed to function in an aqueous environment. Therefore, the key question must be how these components were first assembled and recruited into biology.

Hadean geochemistry can be used inform the selection of likely starting materials and environments, although caution must be exercised because models of this ancient aeon are

\footnotetext{
${ }^{1}$ Department of Chemistry, University College London, 20 Gordon Street, London WC1H OAJ, UK. Correspondence and requests for materials should be addressed to M.W.P. (email: matthew.powner@ucl.ac.uk)
} 
constantly changing. For example, ideas about the composition of the early Earth's atmosphere have radically changed over the last 70 years. The Hadean atmosphere was certainly different from the modern atmosphere: molecular oxygen, for example, is predominantly biogenic on Earth and would not have been present in significant quantities. The Jack Hills zircons provide evidence for liquid water on Earth $4.4 \mathrm{Ga}^{1}$, placing rough constraints on global surface temperatures and, when coupled with predicted solar flux, the atmospheric composition. Accordingly, it has been suggested that the partial pressure of greenhouse gases must have compensated for the lower intensity of solar radiation to maintain liquid water on the early Earth, but the precise composition is open to debate ${ }^{4}$. Initially, a reducing atmosphere with substantial quantities of $\mathrm{CH}_{4}$ was thought to be likely, but this is currently deemed unrealistic due to the rapid destruction of $\mathrm{CH}_{4}$ by UV light ${ }^{5}$, and an atmosphere that contained mostly $\mathrm{N}_{2}$ and $\mathrm{CO}_{2}$ is considered more likely. Experimentally, it has been shown that energetic input (e.g. UV light or electric discharge) into mixtures of $\mathrm{N}_{2}, \mathrm{H}_{2} \mathrm{O}, \mathrm{H}_{2}$ and either $\mathrm{CH}_{4}$ or $\mathrm{CO}_{2}$ produces highly complex mixtures of organic molecules, a very small fraction of which could be considered biologically relevant ${ }^{6}$. Similarly complex mixtures of molecules have been found on carbonaceous meteorites $^{7}$, which suggests that complexity is almost inevitable when enough energy is supplied to simple carbon-containing systems. A more difficult, and likely more important, question is how the prebiotic molecules that are useful to life could be selected from amongst the other, chemically similar, compounds. Improved selectivity in prebiotic reaction pathways can certainly provide part of the answer; physical processes, such as selective crystallisation ${ }^{8}$, must also have been important.

Although the environment from which life first emerged is far from certain, determining the key prebiotic environment(s) that can support the synthesis, purification and accumulation of the essential building blocks of biology can, and should, be a two-way process between mechanistic chemistry and planetary science. Chemical constraints can be used to ascertain the likely geochemical environments of interest. For example, reactions between alcohols and phosphate salts (necessary for the formation of nucleotides) are prohibited in aqueous solution but are facile under dry conditions ${ }^{3}$; as such, these chemical constraints suggest that environments that can support wet-dry cycles (e.g. small ponds) could have been essential during prebiotic nucleotide synthesis. Cross-referencing these chemically constrained conditions with planetary scenarios can further constrain the potential environments or open new avenues of geochemically informed synthesis. However, ultimately all such scenarios could be pre-emptively restrictive until we have more evidence to implicate a particular chemical pathway. The Earth's

a
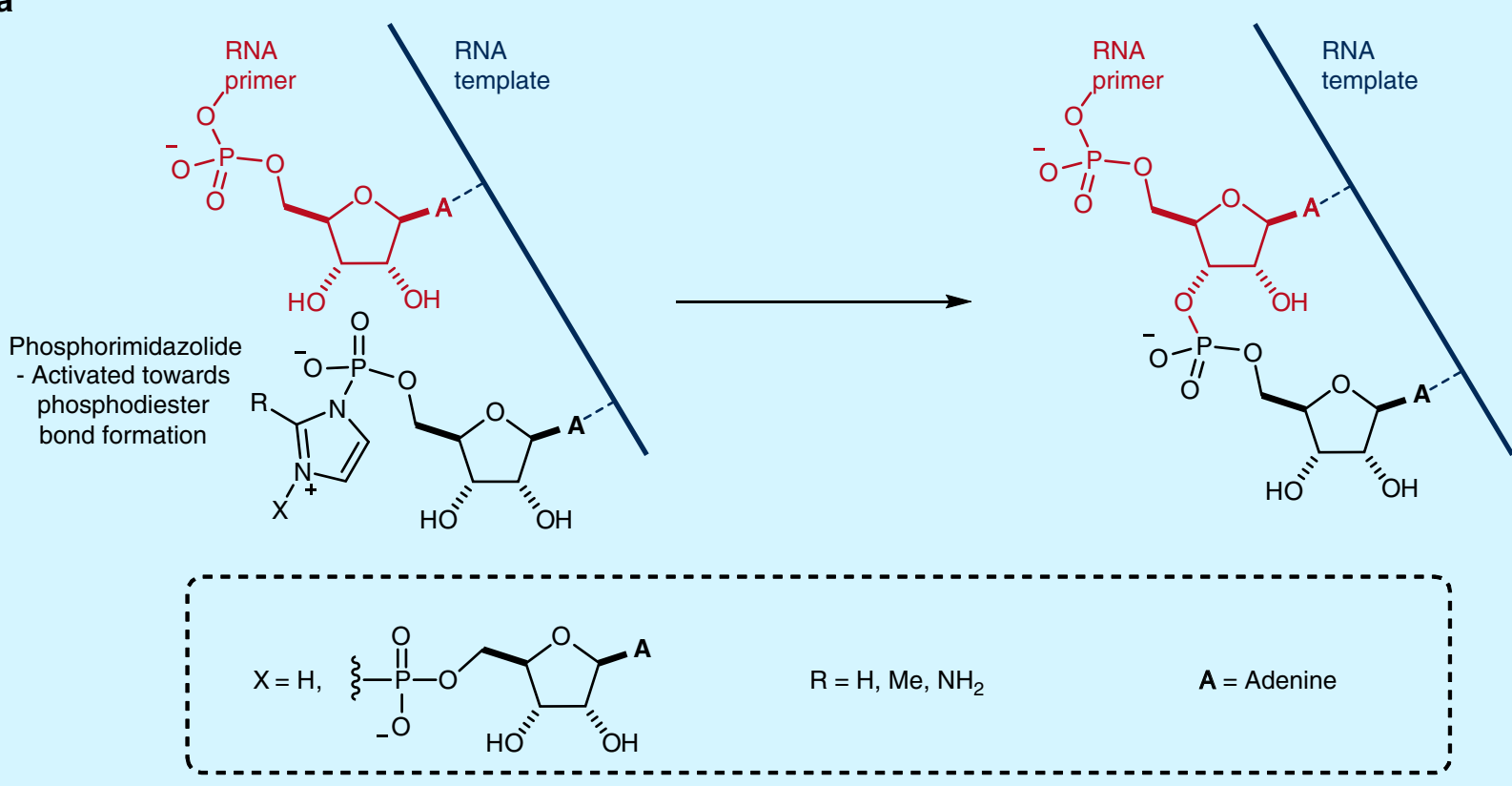

b<smiles>COP(=O)(O)OC[C@H]1O[C@@H](I)[C@@H](O)[C@H]1O</smiles>

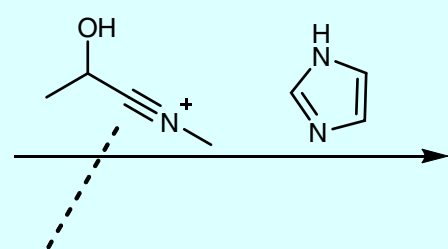

Activating agent

photochemically generated from $\mathrm{Fe}(\mathrm{CN})_{6}, \mathrm{NO}_{2}, \mathrm{MeNH}_{2}$

Fig. 1 Phosphorimidazolide activation of nucleotides. a Phosphorimidazolide mediated non-enzymatic template-directed RNA primer extension. b Prebiotically plausible synthesis of adenosine- $5^{\prime}$-phosphorimidazolide $(R=H)$, using a photochemically generated activating agent 
geochemistry is hugely heterogeneous, with great regional variations across the planet. Locally high concentrations of rare minerals may have been present, particularly given the increased prevalence of meteorite impacts during the Hadean aeon. Stringent restrictions upon which minerals and geochemicals would or would not have been abundant are not necessarily helpful to the question at hand given the huge uncertainty in current models, limited direct evidence of Hadean geochemistry and the unknown role of niche environments during the origin of life.

The uncertainty about the exact conditions required at the origin of life should not be discouraging, nor should it be used to prohibit research. Over time, our models have become more detailed and they, and our concept of what is prebiotic, will continue to evolve. For example, despite the potential importance of trace chemical species in the origins of life, as feedstocks or catalysts, it is difficult to determine their abundance on the early Earth. The rarity of trace species inevitably results in their limited impact on the already scarce rock record. Further work modelling trace species is required. However, importantly, Kepler data suggest that approximately 1 -in-5 stars host rocky planets in their habitable zone $\mathrm{e}^{9}$, and characterising their atmospheres may provide an unrivalled experimental window on the atmospheric composition of early Earth-like planets that can be used to sharpen our models of prebiotic plausibility further. Nevertheless, defining a prescriptive list of chemicals currently thought to have been available on the early Earth would likely be more restrictive than helpful in the long term, as any such list will inevitably change over time. A potentially illuminating case study, that highlights such a change, is the use of phosphorimidazolides as substrates in studies of non-enzymatic RNA replication (Fig. 1a) ${ }^{10,11}$.

These activated nucleotides react with a short strand of RNA (a primer, bound to templating complementary RNA) much more facilely than (biological) nucleoside triphosphates. Researchers studying phosphorimidazolides have typically presented them as useful models to probe the difficult problem of non-enzymatic RNA replication, rather than as prebiotically plausible substrates. However, imidazolide studies have provided great mechanistic insights into abiotic nucleic acid replication over the past 50 years, and this has ultimately prompted the discovery of a prebiotically plausible route for their formation (Fig. 1b) ${ }^{12}$. Recently, these studies also led to the discovery that, out of many different imidazole-type molecules, 2-aminoimidazole (2AI) is the superior activating agent ${ }^{13}$. Interestingly, $2 \mathrm{AI}$ can be made through a prebiotically plausible reaction which is remarkably similar to the synthesis of two other molecules that have been implicated in prebiotic nucleotide chemistry: 2-aminooxazole (2AO) and 2-aminothiazole (2AT) (Fig. 2). 2AO provides a robust and selective route to nucleotides which bypasses free ribose (Fig. 2, pink) ${ }^{14,15}$, while $\mathbf{2 A T}$ is able to chaperone this synthesis via selective time-resolved crystallisation of the required aldehydes, GC and GA, from highly complex sugar mixtures, and dynamically resolving C3 aldose sugar GA from its more thermodynamically stable ketose isomer DHA (Fig. 2, yellow) ${ }^{8}$. Structurally, these three azole molecules are remarkably similar and could all share a common origin from cyanamide (1). They<smiles>Nc1nccs1</smiles><smiles>O=CCO</smiles>
2AT<smiles>CSCC=O</smiles>

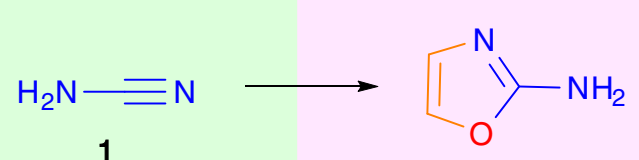

1<smiles>NCC=O</smiles><smiles>Nc1ncc[nH]1</smiles>

2AI
$2 \mathrm{AO}$

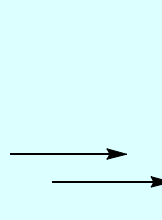
$\mathrm{GC}$<smiles>CN=C(N)OC</smiles><smiles>O=C(CO)CO</smiles><smiles>[3H]C</smiles><smiles>O=CC(O)CO</smiles>

GA

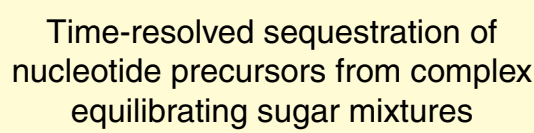

$\longrightarrow$<smiles>NC1=NC2OC(CO)[C@H](O)C2O1</smiles><smiles>[Y][V]</smiles>

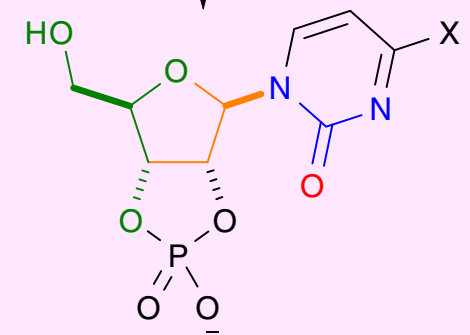

Fig. 2 The roles of 2-aminoazoles in prebiotically plausible nucleotide chemistry. Green: Divergent synthesis of 2-aminothiazole (2AT), 2-aminooxazole (2AO) and 2-aminoimidazole (2AT) from cyanamide (1). Yellow: Crystallisation-controlled aldehyde sequestration by 2-aminothiazole (2AT) giving access to pure glycolaldehyde (GC) and glyceraldehyde (GA), in the order required for selective nucleotide synthesis, from equilibrating sugar mixtures. Pink: Prebiotically plausible pyrimidine nucleotide synthesis. Cytidine $\mathrm{X}=\mathrm{NH}_{2}$, uridine $\mathrm{X}=\mathrm{OH}$. Blue: Nucleoside-5'-phosphorimidazolide activation with 2- 
play three distinct roles in facilitating prebiotically plausible nucleotide chemistry, but none of these would have been considered prebiotic molecules 15 years ago. It is possible that these imidazolide, and the prebiotically plausible pathways they implicate, will be superseded by more efficient, more robust and more accurate prebiotic pathways to RNA, but by studying them invaluable information about the chemical reactivity of simple aqueous systems that can selectively build nucleic acids has been discovered. Had researchers ignored (or been dissuaded from investigating) these systems on the grounds that they were thought not to be prebiotically plausible, these insights may have been missed.

Ultimately, until several routes have been found by which simple abiotic molecules can be transformed into systems on which Darwinian evolution can act, discussions about which reactions are more (or less) prebiotically plausible than others are simply less instructive than investigating chemical reactivity. By limiting the scope of our investigations too strictly, we will always risk missing crucial aspects of the story as it unfolds.

Received: 23 September 2018 Accepted: 19 October 2018

Published online: 12 December 2018

\section{References}

1. Watson, E. B. Zircon thermometer reveals minimum melting conditions on earliest earth. Science 308, 841-844 (2005).

2. Theobald, D. L. A formal test of the theory of universal common ancestry. Nature 465, 219-222 (2010).

3. Orgel, L. E. Prebiotic chemistry and the origin of the RNA world. Crit. Rev. Biochem. Mol. Biol. 39, 99-123 (2004).

4. Zahnle, K., Schaefer, L. \& Fegley, B. Earth's earliest atmospheres. Cold Spring Harb. Perspect. Biol. 2, a004895 (2010).

5. Kasting, J. F., Zahnle, K. J. \& Walker, J. C. G. Photochemistry of methane in the Earth's early atmosphere. Precambrian Res. 20, 121-148 (1983).

6. Miller, S. L. \& Schlesinger, G. The atmosphere of the primitive earth and the prebiotic synthesis of organic compounds. Adv. Space Res. 3, 47-53 (1983).

7. Sephton, M. A. Organic compounds in carbonaceous meteorites. Nat. Prod. Rep. 19, 292-311 (2002).

8. Islam, S., Bučar, D.-K. \& Powner, M. W. Prebiotic selection and assembly of proteinogenic amino acids and natural nucleotides from complex mixtures. Nat. Chem. 9, 584-589 (2017).
9. Kaltenegger, L. How to characterize habitable worlds and signs of life. Annu. Rev. Astron. Astrophys. 55, 433-485 (2017).

10. Weimann, B. J., Lohrmann, R., Orgel, L. E., Schneider-Bernloehr, H. \& Sulston, J. E. Template-directed synthesis with adenosine-5'-phosphorimidazolide. Science 161, 387-387 (1968).

11. Zhang, W., Walton, T., Li, L. \& Szostak, J. W. Crystallographic observation of nonenzymatic RNA primer extension. eLife 7, 1-15 (2018).

12. Mariani, A., Russell, D. A., Javelle, T. \& Sutherland, J. D. A light-releasable potentially prebiotic nucleotide activating agent. J. Am. Chem. Soc. 140, 8657-8661 (2018)

13. Fahrenbach, A. C. et al. Common and potentially prebiotic origin for precursors of nucleotide synthesis and activation. J. Am. Chem. Soc. 139, 8780-8783 (2017).

14. Powner, M. W., Gerland, B. \& Sutherland, J. D. Synthesis of activated pyrimidine ribonucleotides in prebiotically plausible conditions. Nature $\mathbf{4 5 9}$, 239-242 (2009)

15. Stairs, S. et al. Divergent prebiotic synthesis of pyrimidine and 8-oxo-purine ribonucleotides. Nat. Commun. 8, 15270 (2017).

\section{Author contributions}

M.W.P. and D.W. wrote the paper.

\section{Additional information}

Competing interests: The authors declare no competing interests.

Reprints and permission information is available online at http://npg.nature.com/ reprintsandpermissions/

Publisher's note: Springer Nature remains neutral with regard to jurisdictional claims in published maps and institutional affiliations.

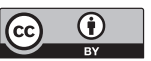

Open Access This article is licensed under a Creative Commons Attribution 4.0 International License, which permits use, sharing, adaptation, distribution and reproduction in any medium or format, as long as you give appropriate credit to the original author(s) and the source, provide a link to the Creative Commons license, and indicate if changes were made. The images or other third party material in this article are included in the article's Creative Commons license, unless indicated otherwise in a credit line to the material. If material is not included in the article's Creative Commons license and your intended use is not permitted by statutory regulation or exceeds the permitted use, you will need to obtain permission directly from the copyright holder. To view a copy of this license, visit http://creativecommons.org/ licenses/by/4.0/.

(C) The Author(s) 2018 